\title{
Squamous Cell Carcinoma Antigen-encoding Genes SERPINB3/B4 as Potentially Useful Markers for the Stratification of HNSCC Tumours
}

\author{
ZUZANA SAIDAK $^{1,2}$, MONY CHENDA MORISSE ${ }^{2,3}$, DENIS CHATELAIN ${ }^{2,4}$, \\ CHLOÉ SAUZAY $^{2,3}$, ALINE HOUESSINON ${ }^{2,5}$, NELLY GUILAIN ${ }^{4}$, MARION SOYEZ $^{3}$, \\ BRUNO CHAUFFERT ${ }^{2,5}$, STÉPHANIE DAKPÉ ${ }^{2,6}$ and ANTOINE GALMICHE ${ }^{2,3}$ \\ ${ }^{1}$ Department of Molecular Oncobiology, CHU Sud, Amiens, France; \\ ${ }^{2}$ CHIMERE team, University of Picardie Jules Verne, Amiens, France; \\ ${ }^{3}$ Department of Biochemistry, CHU Sud, Amiens, France; \\ ${ }^{4}$ Department of Pathology, CHU Nord, Amiens, France; \\ ${ }^{5}$ Department of Medical Oncology, CHU Sud, Amiens, France; \\ ${ }^{6}$ Department of Maxillofacial Surgery, CHU Sud, Amiens, France
}

\begin{abstract}
Background/Aim: The squamous cell carcinoma antigen (SCCA), encoded by the genes SERPINB3/B4, is a tumour marker produced by head and neck squamous cell carcinoma (HNSCC). We aimed to examine SERPINB3/B4 $m R N A$ levels and its clinical significance in the therapeutic context. Materials and Methods: We retrieved mRNA expression levels, clinical, pathological and genomic data for $520 \mathrm{HNSCC}$ from The Cancer Genome Atlas (TCGA). Results: HNSCC tumours express high levels of SERPINB3/B4 mRNA. SERPINB3 expression differs depending on Human papillomavirus (HPV) infection status, primary tumour location, grade and differentiation, extension to lymph nodes and extracapsular spread. Interestingly, we observed an association between SERPINB3/B4 and the presence of tumour immune infiltrate as well as the expression of the immune checkpoint regulators $P D$ L1/PD-L2 that depended on HPV status. Conclusion: Our findings point to potential interest of SERPINB3/B4 for the stratification of HNSCC patients in the therapeutic context.
\end{abstract}

Head and neck squamous cell carcinoma (HNSCC) is the 6th most frequent type of solid tumour in the world. Alcohol and

This article is freely accessible online.

Correspondence to: Dr. Antoine Galmiche, Department of Biochemistry, Human Biology Center (CBH), CHU Amiens Sud, Avenue Laennec, 80054 Amiens Cedex, France. Tel: +33 0322087017 , Fax: +33 0322087026, e-mail: Galmiche.Antoine@chu-amiens.fr

Key Words: SERPINB3/B4, head \& neck squamous cell carcinoma, TCGA. tobacco use are two important risk factors for these tumours $(1,2)$. Human papillomavirus (HPV) infection is another important risk factor that is responsible for the recent increase in the incidence of oropharynx tumours in economically developed countries (1, 2). Surgery, radiotherapy, chemotherapy and cetuximab, a monoclonal antibody targeting the epidermal growth factor receptor (EGFR) constitute the mainstay of the treatment of HNSCC (3-5). Despite these therapeutic options, the natural history of HNSCC is marked by a high risk of recurrence and a poor prognosis $(1,2)$. Recently, targeting the immune checkpoint regulator programmed cell death-1 (PD-1) with monoclonal antibodies, such as pembrolizumab and nivolumab, proved efficacious in the treatment of recurrent/metastatic HNSCC (6-8). Nivolumab is now approved by the FDA for the treatment of patients with recurrent or metastatic HNSCC that fail to respond to platinum-based therapy. While clinical trials are ongoing to define the optimal strategy involving these new molecules, it is clear at this stage that biological markers are needed to adjust the best therapeutic strategy for each individual patient.

The tumour antigen SCCA (Squamous Cell Carcinoma Antigen) was initially isolated from squamous cell carcinoma originating from the cervix (9). Following its discovery, SCCA has been detected in multiple types of primary tumours, and in the serum of cancer patients, making it a protein marker of potential clinical interest in HNSCC (9). In the human genome, SCCA is encoded by two genes (SERPINB3/SERPINB4) located on the long arm of chromosome 18 (18q21). The corresponding protein products are two highly homologous protein members of the Serpin family of Ser protease inhibitors that function as suicide substrates for cellular proteases (9). These two proteins are 
Table I. Clinical characteristics of HNSCC patients $(n=520)$.

\begin{tabular}{ll}
\hline Gender & Male $(\mathrm{n}=382) ;$ Female $(\mathrm{n}=135) ; \mathrm{N} / \mathrm{A}(\mathrm{n}=3)$ \\
Age (median, years) & 61 \\
HPV status & HPV-negative $(\mathrm{n}=72) ;$ HPV-positive $(\mathrm{n}=37) ;$ N/A $(\mathrm{n}=412)$ \\
Primary tumour location & Larynx $(\mathrm{n}=116)$ \\
& Oral Cavity $(\mathrm{n}=313)$ \\
& Oropharynx $(\mathrm{n}=78)$ \\
& Hypopharynx $(\mathrm{n}=10)$ \\
& N/A $(\mathrm{n}=3)$ \\
& T0 $(\mathrm{n}=1), \mathrm{T} 1(\mathrm{n}=48), \mathrm{T} 2(\mathrm{n}=134), \mathrm{T} 3(\mathrm{n}=99), \mathrm{T} 4 / \mathrm{a} / \mathrm{b}(\mathrm{n}=173), \mathrm{TX}(\mathrm{n}=39), \mathrm{N} / \mathrm{A}(\mathrm{n}=26)$ \\
TNM & N0 $(\mathrm{n}=176), \mathrm{N} 1(\mathrm{n}=67), \mathrm{N} 2 / \mathrm{a} / \mathrm{b} / \mathrm{c}(\mathrm{n}=166), \mathrm{N} 3(\mathrm{n}=8), \mathrm{NX}(\mathrm{n}=75), \mathrm{N} / \mathrm{A}(\mathrm{n}=28)$ \\
& M0 $(\mathrm{n}=184), \mathrm{M} 1(\mathrm{n}=1), \mathrm{MX}(\mathrm{n}=61), \mathrm{N} / \mathrm{A}(\mathrm{n}=274)$ \\
\hline
\end{tabular}

HPV status based on p16 labelling by immunohistochemistry; TNM staging according to the American Joint Committee on Cancer (AJCC).

both localized in the cytosol, but are likely active against distinct intracellular proteases: SCCA1, encoded by SERPINB3, is able to inhibit lysosomal cathepsins (isoforms $\mathrm{S}, \mathrm{L}$, and K), while SCCA2, encoded by SERPINB4 inhibits cathepsin G (10). Early studies performed in HNSCC cells have shown that SCCA1 regulates cell death associated with protease activation, for example upon lysosomal leakage (1113). In agreement with the widely-accepted view that matrix invasion depends on functional proteases, overexpression of SERPINB3 was also found to reduce cancer cell invasion in matrigel assays (14).

Recent studies performed on multiple types of tumours have unveiled new functional consequences of the neutralization of cellular proteases by SERPINB3/B4 (15-16). In epithelial cells from various types of adenocarcinomas, the introduction of an activated RAS oncogene was found to induce SERPINB3/SERPINB4 expression, leading to endoplasmic reticulum stress and a cellular homeostatic response known as the unfolded protein response (UPR) (17, 18). Through its ability to neutralize intracellular proteases and to induce the UPR, SERPINB3 may contribute to the installation of a pro-inflammatory phenotype characterized by high expression of the cytokine interleukin-6 (IL6) in colorectal and pancreatic adenocarcinomas (15). More recently, mutations of the SERPINB3/B4 genes were found in melanoma patients receiving the immune checkpoint inhibitor ipilimumab (19). This observation opens up the possibility that SERPINB3/B4 expression might be a determinant of tumour immunogenicity in melanoma in the therapeutic context (19).

While these new studies unveil a potential role of SERPINB3/B4 as a determinant of tumour progression and response to various treatments, no study to date has examined this possibility in HNSCC. In the present study, we aimed to examine the clinical and pathological significance of SERPINB3/SERPINB4 expression in HNSCC, using data from The Cancer Genome Atlas (TCGA) (20).

\section{Materials and Methods}

TCGA datasets. Patient clinical, pathological data and RNA-seq expression values (RNA SeqV2 RSEM), were retrieved from TCGA in September 2017, using Cbioportal at: http://cbioportal.org $(21,22)$. Altogether, 24 tumour datasets were analysed, representing a total of 10,663 patients and 520 HNSCC (Table I). Gene expression was normalised to TBP (TATA-box binding protein) expression as indicated.

Gene Ontology (GO) analysis. We used the PANTHER classification system (http://pantherdb.org/) to perform a statistical overrepresentation test. The enrichment of Gene Ontology (GO) terms in our gene set was compared to the whole Homo Sapiens genome (23), with Bonferroni correction applied.

IMMUNE score analysis. The IMMUNE score was used to evaluate tumour purity, i.e. the infiltration levels of immune cells based on gene expression data (24).

Statistical analyses. Pearson correlation, Cox regression, KruskalWallis and Wilcoxon tests were used as indicated and were all performed using R (packages Hmisc and Survival).

\section{Results}

High expression of SERPINB3/SERPINB4 in HNSCC. In order to examine the expression levels of SERPINB3 and SERPINB4 in various types of tumours, we performed an analysis of their mRNA expression levels, normalized to TBP (TATA box-Binding protein) mRNA expression (Figure 1). SERPINB3 was found to be expressed at significant levels in a broad variety of tumours, with the highest expression levels in cervical, head and neck and lung squamous tumours (Figure 1A). The same pattern of expression was detected with SERPINB4, even though the expression levels of SERPINB4 were on average 4x lower than those of SERPINB3 (Figure 1B). In HNSCC tumours, the expression levels of SERPINB3 were highly correlated with those of SERPINB4 $\left(\mathrm{R}^{2}=0.92, p<0.001\right)$ (Figure $\left.1 \mathrm{C}\right)$. 

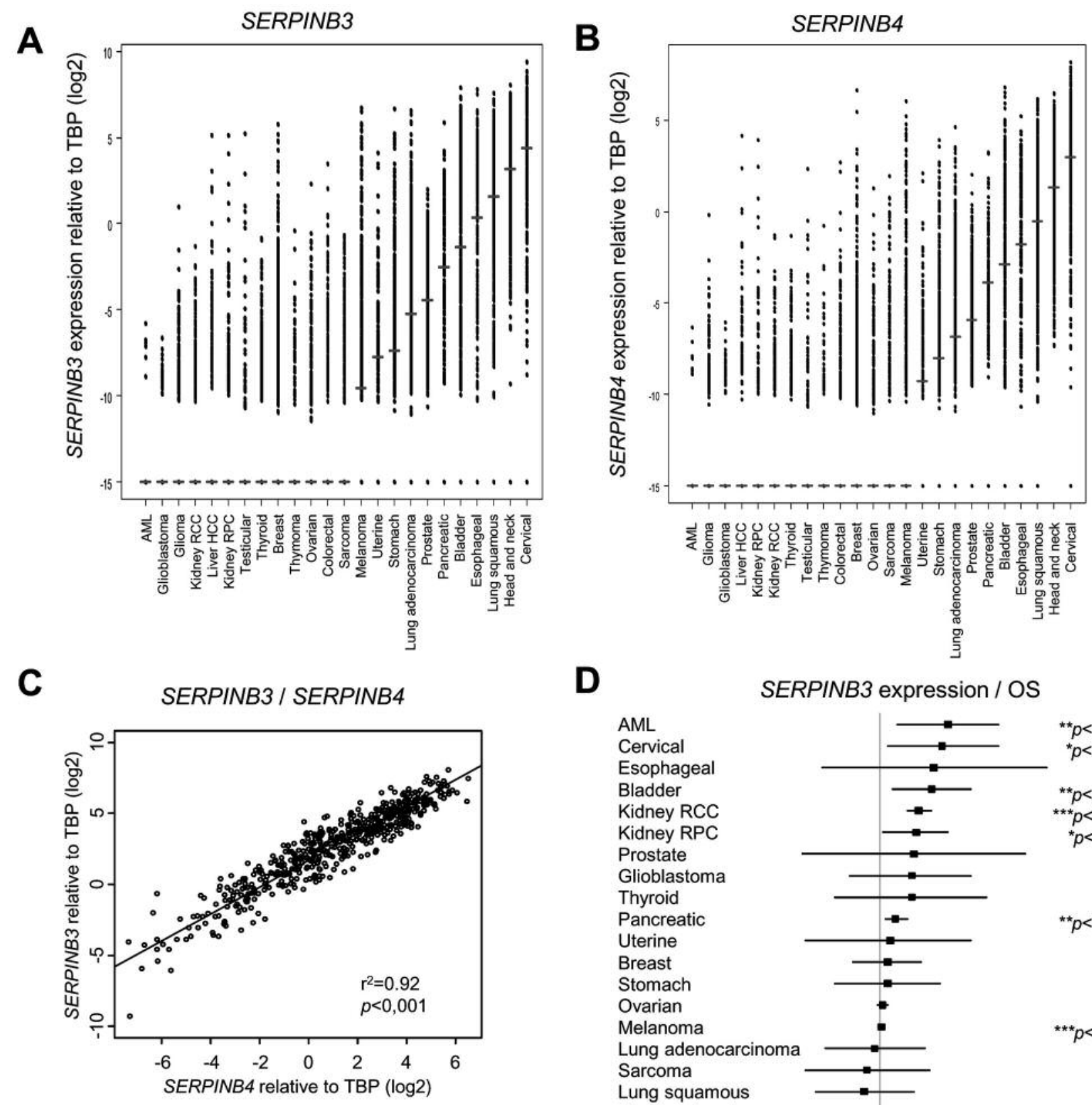

D

AML

Cervical

Esophageal

Bladder

Kidney RCC

Kidney RPC

Prostate

Glioblastoma

Thyroid

Pancreatic

Uterine

Breast

Stomach

Ovarian

Melanoma

Lung adenocarcinoma

Sarcoma

Lung squamous

Head and neck

Glioma
SERPINB3 expression / OS
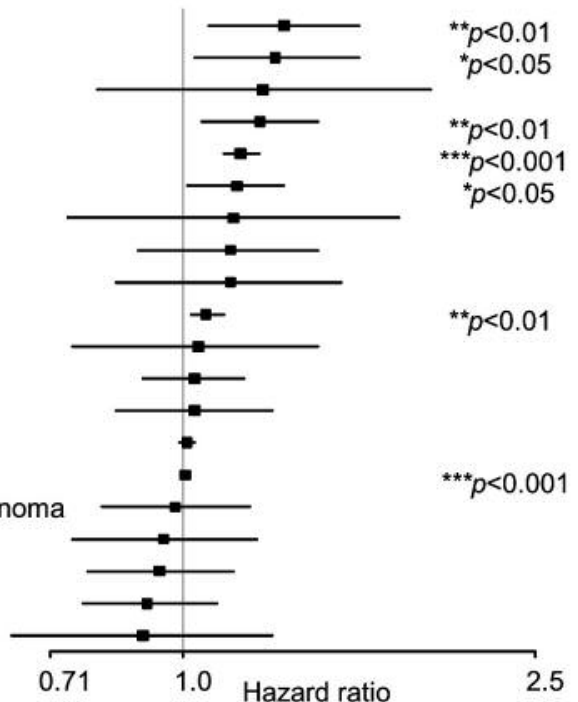

Figure 1. Expression of SERPINB3 and SERPINB4 in different human tumours. A, B. Each data point represents the expression levels of SERPINB3 (A) or SERPINB4 (B) as log2(RSEM values normalized to TBP) in one tumour. Horizontal lines indicate the median expression values. Samples with zero expression are plotted on the bottom of the y axis. C. Pearson correlation between SERPINB3 and SERPINB4 expression (log2(RSEM values normalized to TBP)) in HNSCC (Pearson $\left.r^{2}=0.92, p<0.001\right) D$. A forest plot showing the hazard ratios and 95\% confidence intervals for overall survival for each cancer type, with respect to SERPINB3 expression (Cox proportional hazards regression model). *p<0.05, **p<0.01,***p<0.001.

We therefore limited our subsequent analyses to the regulation of SERPINB3. To address the possibility that the expression of SCCA might have a prognostic value, we calculated the hazard ratio for overall survival across the different types of primary tumours included in this analysis (Figure 1D). In cervical tumours, expression of SERPINB3 was associated with a hazard ratio $>1$, representing a significantly reduced OS and an unfavorable outcome 

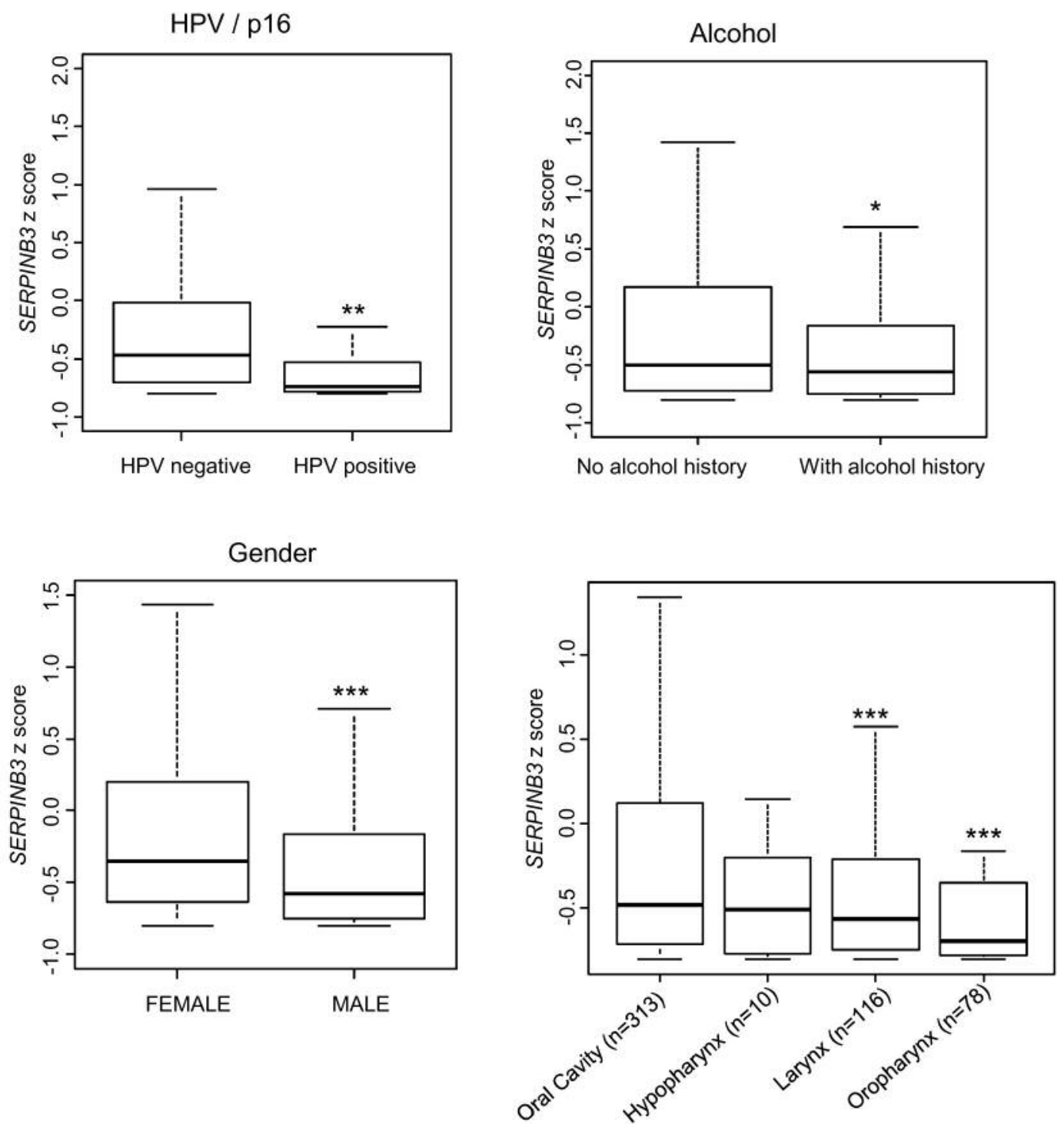

Figure 2. Clinical correlates of SERPINB3 in HNSCC. Boxplots showing SERPINB3 expression (z scores) in HNSCC patients based on HPV status (p16) $(H P V+n=72, H P V-n=37)$, history of alcohol use (with alcohol history $n=346$, no alcohol history $n=160$ ) and gender (male $n=382$, female $n=135)$. The lower panel is an analysis of SERPINB3 expression depending on the location of the primary tumour. The groups were compared to the highest SERPINB3 expressor "Oral cavity". Wilcoxon test was used for statistical analyses. ${ }^{*} p<0.05, * * p<0.01, * * * p<0.001$.

(hazard ratio $=1.27, p<0.05$ ). No significant effect of the SERPINB3 status was found for HNSCC patients.

SERPINB3 expression is associated with clinical and pathological characteristics in HNSCC. The levels of SERPINB3 were significantly higher in the population of patients with a HPV-negative status (as determined from the expression of the viral oncoprotein p16) (Figure 2). A modest, yet significant link to alcohol exposure was found: HNSCC tumours arising in a context of alcohol intake expressed slightly lower levels of SERPINB3 (Figure 2). No link was found to smoking (data not shown). Male patients expressed lower levels of SERPINB3 mRNA compared to female patients (Figure 2). Expression levels of SERPINB3 in HNSCC differed according to the localization of the primary tumour (Figure 2). Tumours arising in the oral cavity had significantly higher SERPINB3 expression levels compared to other locations, such as the larynx and oropharynx (Figure 2).

We examined the association of SERPINB3 expression levels with HNSCC staging, as defined by the American Joint Committee on Cancer Code (AJCC). We found no significant difference in SERPINB3 expression levels according to tumour volume (Figure 3A). Extension to lymph nodes and 

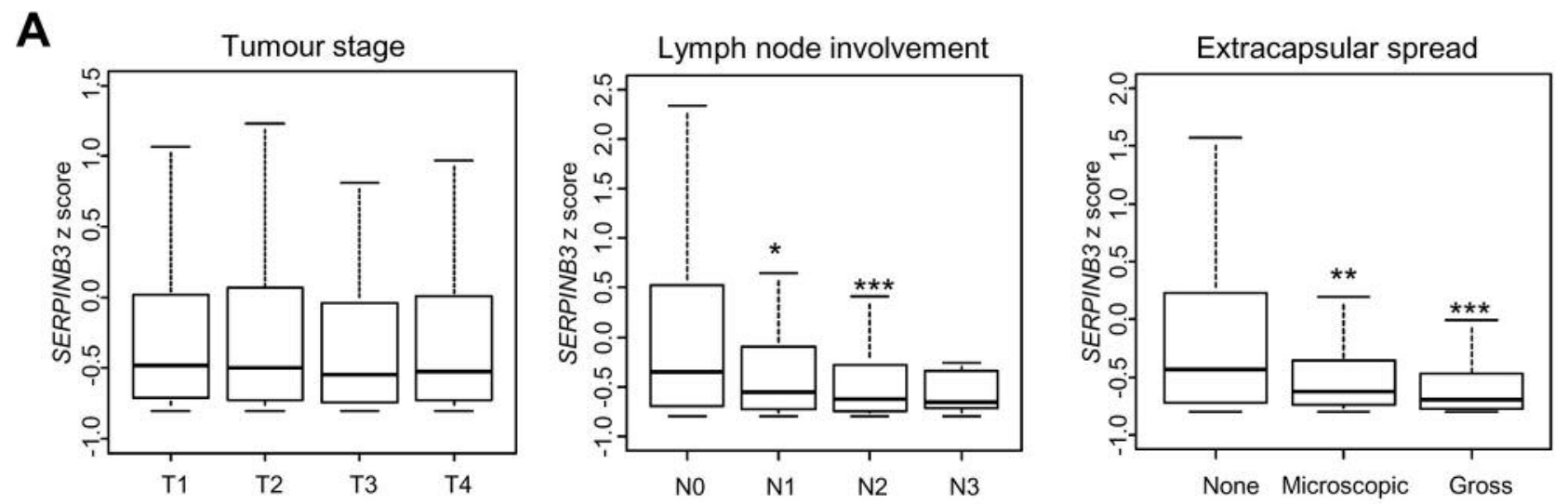

B
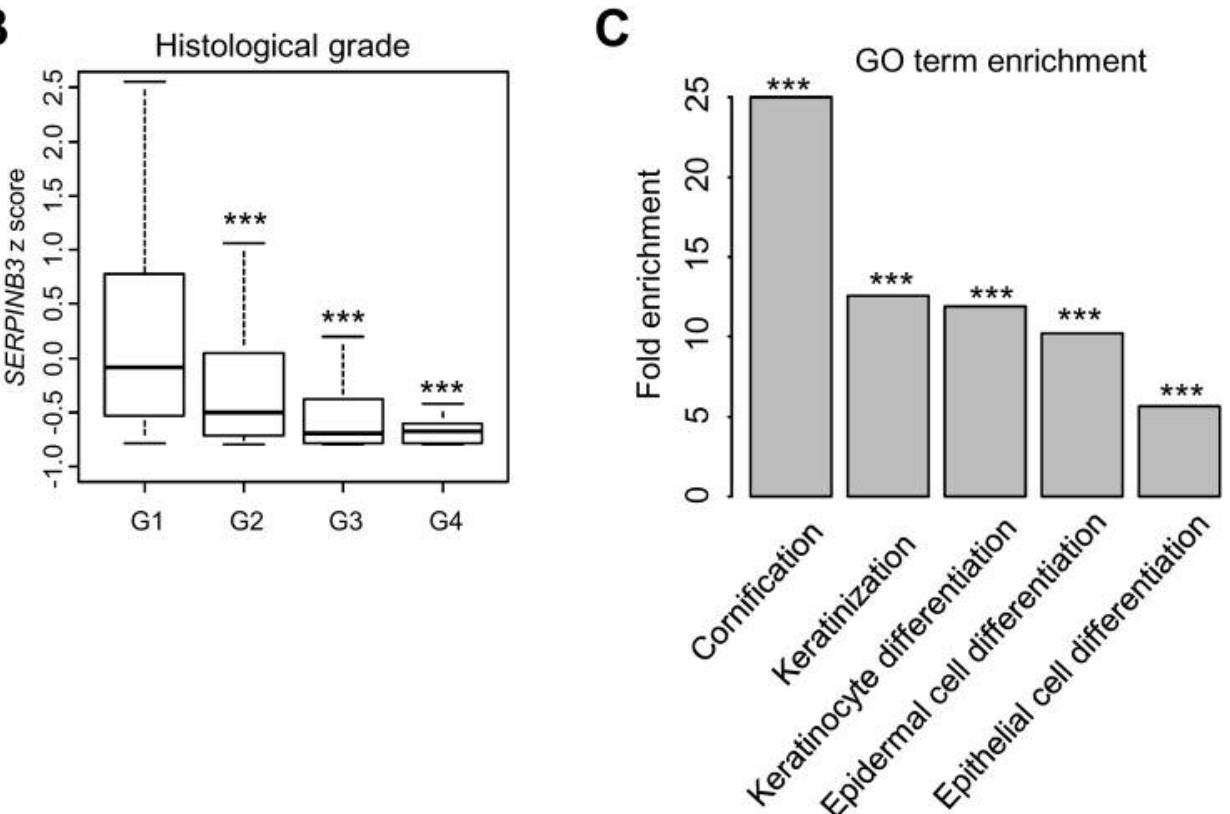

Figure 3. Pathological correlates of SERPINB3 in HNSCC. A. Boxplots representing SERPINB3 expression (z score) in HNSCC tumours of different stage $(n=520)$. Tumour stage and lymph node involvement are given according to the American Joint Committee on Cancer. T: T1 ( $n=48), T 2$ $(n=134), T 3(n=99), T 4(n=173) . N: N O(n=176), N 1 \quad(n=67), N 2(n=166), N 3(n=8)$. Extracapsular spread was estimated as absent $(n=243)$, microscopic $(n=74)$ or gross $(n=36)$. B. Boxplots of SERPINB3 expression (z scores) in HNSCC tumours according to histological grade (G1 to $G 4$, with G1 representing well differentiated tumors and G3/4 poorly differentiated tumors): G1 ( $n=61)$, G2 $(n=302), G 3(n=125)$, G4 ( $n=7)$. Wilcoxon test was used for statistical analyses compared to control. ${ }^{*} p<0.05, * * p<0.01, * * * p<0.001$. C. A Gene ontology (GO) analysis was performed on the panel of genes that correlate with the expression of SERPINB3 in HNSCC and cervical tumours $(n=115)$. For each indicated GO term, we present a calculation of the fold-enrichment. $* * * p<0.001$.

extracapsular spread were however associated with lower expression levels of SERPINB3 (Figure 3A). HNSCC tumours of high grade (G2, G3 and G4) had significantly lower expression levels of SERPINB3 compared to those that were well-differentiated (G1) (Figure 3B). To support this observation suggesting that SERPINB3 is a marker of epidermal differentiation, we performed a gene ontology
(GO) analysis of the genes that correlated with SERPINB3 expression in SCC (Figure 3C). We identified 115 genes that correlated with SERPINB3 mRNA in HNSCC and cervical cancer (Pearson $\mathrm{R}^{2}>0.30$ ). The GO term "cornification" (accession: GO:0070268) was the most significantly enriched biological process (25-fold enrichment, $p<0.001$ ), followed by "keratinization" (GO:0031424, 12.5-fold enrichment, 


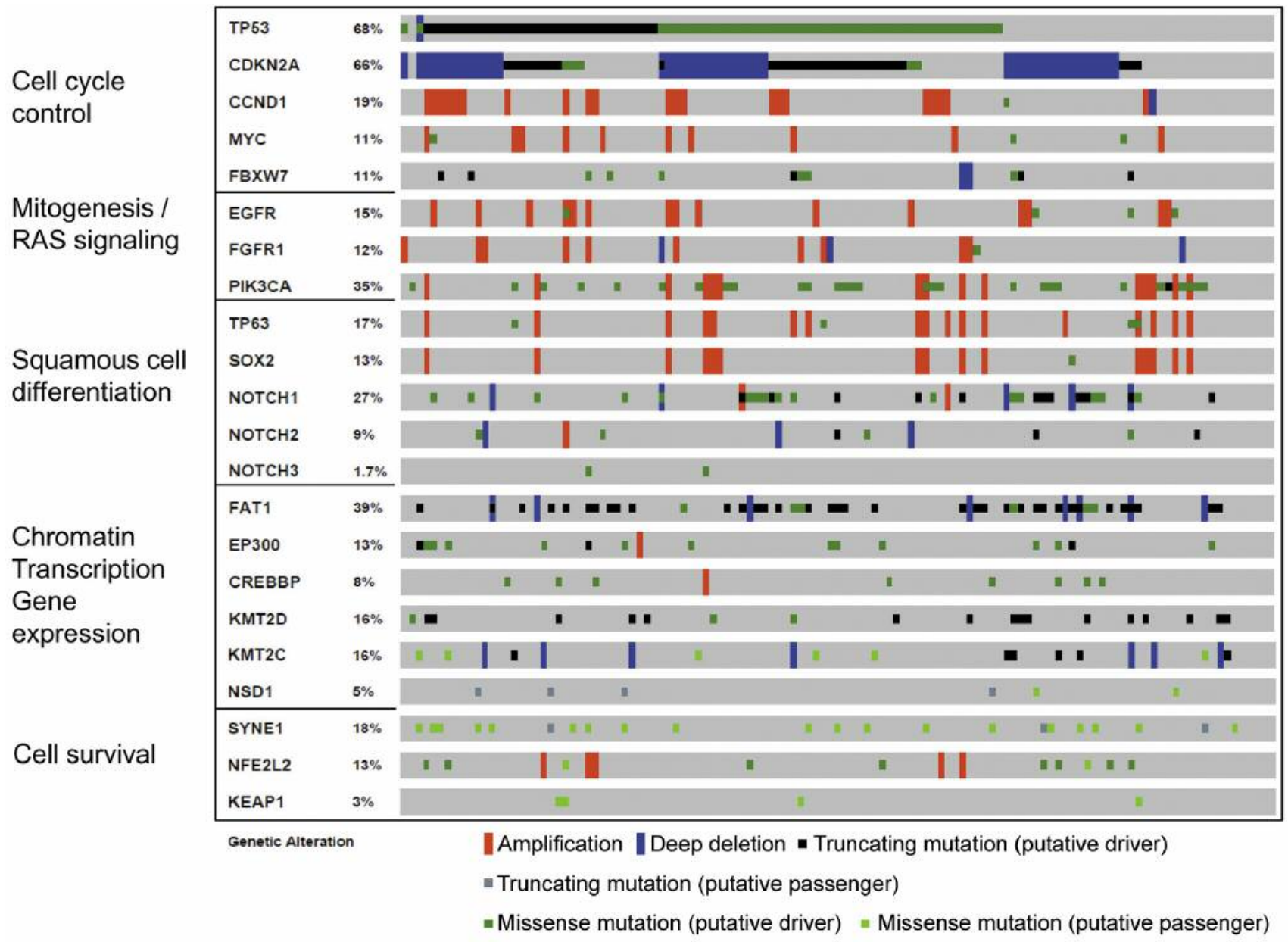

Figure 4. Genetic profile of SERPINB3 expressing HNSCC. An ONCOPRINT representation of gene alterations most commonly found in HNSCC tumours with high expression levels of SERPINB3 $(z>0, n=119)$. For each gene, we present a summary of the main types of alterations (amplification, deletion, missense or truncating mutations, putative drivers or passenger mutations). The genes are classified according to their main cellular function, as previously reported by Dotto et al. (25).

$p<0.001)$, "keratinocyte differentiation" (GO:0030216, 11.9fold enrichment, $p<0.001)$, "epidermal cell differentiation" (GO:009913, 10.2-fold enrichment, $p<0.001$ ) and "epithelial cell differentiation" (GO:0030855, 5.7-fold enrichment, $p<0.001$ ) (Figure 3C). We concluded that SERPINB3 mRNA is a marker of epithelial differentiation in HNSCC.

SERPINB3 as a potential marker of tumour immunogenicity in HNSCC. We examined the possibility that SERPINB3 expression might be related to specific genomic events in HNSCC tumours $(20,25)$. An Oncoprint representation, analysing the genomic events in HNSCC tumours with a positive z score for SERPINB3 mRNA was constructed (Figure 4). This analysis revealed the lack of association between SERPINB 3 overexpression and the presence of gene alterations in the pathways identified as being essential in
SCC (25): cell cycle control (TP53, CDKN2, CCND1, MYC, $F B X W 7$ ), mitogenesis and RAS signaling (EGFR, FGFRl, PIK3CA), squamous cell differentiation (TP63, SOX2, NOTCH1/2/3), chromatin and transcription/gene regulation (FAT1, EP300, CREBBP, KMT2D, KMT2C, NSD1), cell survival (SYNE1, NF2L2, KEPA1) (Figure 4). We also explored the possibility that SERPINB3 status may be associated with tumour mutational burden, by comparing SERPINB3 mRNA expression with the number of somatic mutations in HNSCC tumours. No association was seen between these two parameters (data not shown).

To address the possibility that SERPINB3/B4 could be a determinant of immune response during HNSCC progression and therapeutic response, we turned our attention to three biological parameters: i) the level of tumour immune infiltration; ii) the expression of the immune checkpoint 

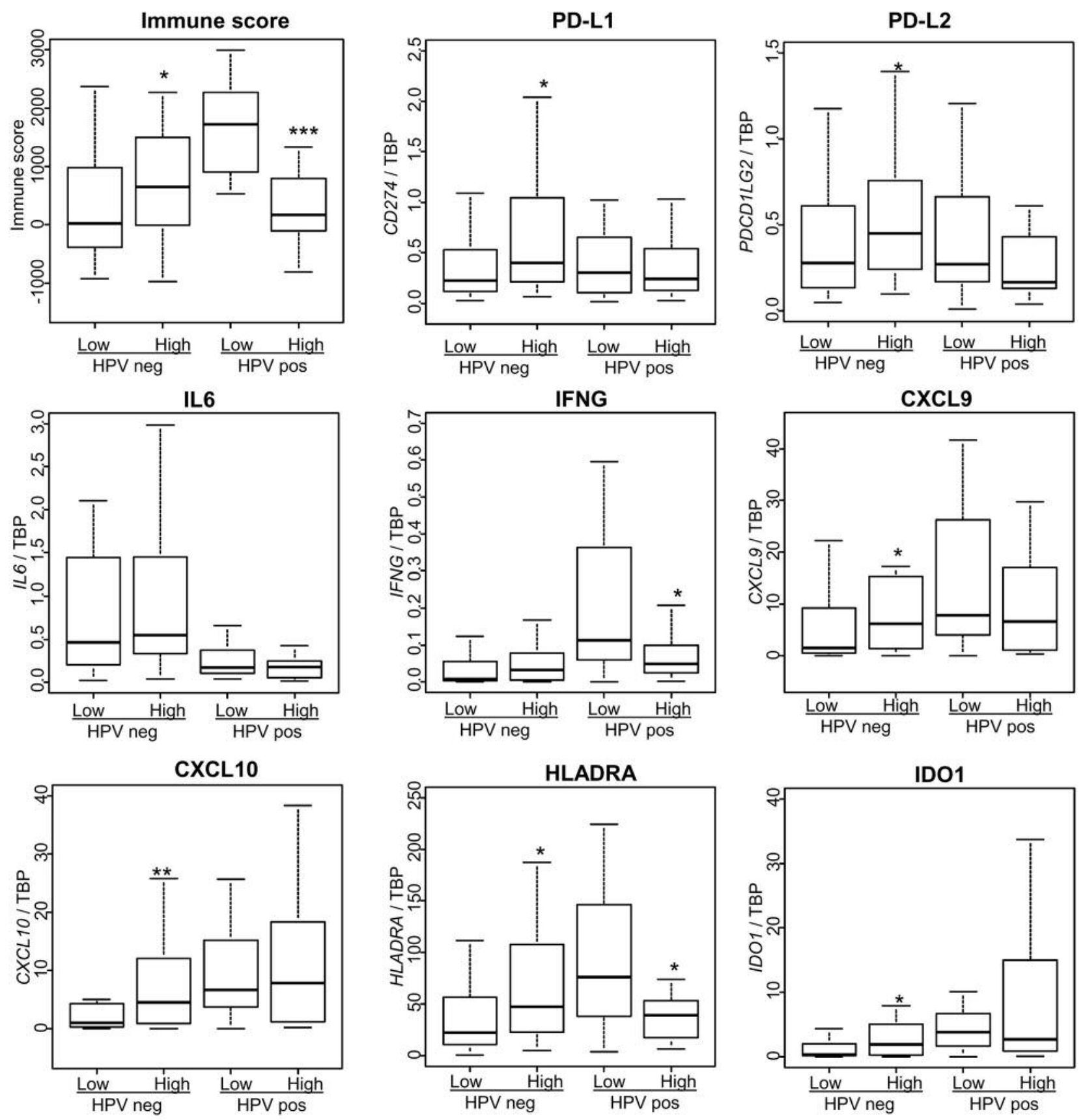

Figure 5. SERPINB3 status as a potential determinant of tumour immunogenicity. The IMMUNE score, representing tumour immune cell infiltration, was calculated using the ESTIMATE algorithm comparing HNSCC tumors with high SERPINB3 expression (above the median) to tumours with low SERPINB3 expression (below the median) in patients stratified by HPV status ( $p 16)(H P V+n=72, H P V-n=37)$. The four groups were tested for their expression of the genes encoding the immune checkpoint modulators PD-L1 (CD274) and PD-L2 (PDCD1LG2) and those associated with the inflammatory and immune responses (IL6, IFNG, CXCL9, CXCL10, HLADRA and IDO1). Wilcoxon test was used to compare the high- and low-expression groups. $* p<0.05, * * p<0.01, * * * p<0.001$.

modulators PD-L1/PD-L2; iii) the local production of immune cytokines (26). HNSCC patients were stratified according to their HPV status, and we used the IMMUNE score, reported by Yoshihara et al. (24) to analyse the levels of tumour immune infiltration (Figure 5). We observed that HPVnegative tumours with high SERPINB3 (above median) had a significantly higher IMMUNE score compared to HPVnegative tumours with low SERPINB3 (below median). An 
opposite effect was found with HPV-positive tumours that were characterized by a higher IMMUNE score compared to those that were HPV-negative: in HPV-positive tumours, overexpression of SERPINB3 was associated with a significantly reduced $1 M M U N E$ score (Figure 5). Interestingly, HPV-negative tumours with high levels of SERPINB3 were also found to express PD-L1 (CD274) and PD-L2 (PDCD1LG2) mRNA at higher levels than those with low levels of SERPINB3, an effect that was not seen in HPVpositive tumours (Figure 5). Finally, we examined the mRNA expression levels of the cytokines interleukin-6 (IL6) and interferon- $\gamma(I F N G)$, as well as the Interferon- $\gamma$ gene signature recently reported by Chow et al. in HNSCC (27). High levels of SERPINB3 were not significantly associated with higher levels of IL6 or IFNG mRNA, but there was a positive association with the IFNG signature (CXCL9, CXCL10, HLADRA, IDO1) in HPV-negative tumours (Figure 5).

\section{Discussion}

Analysis of genetic alterations and gene expression profiles holds potential for the stratification of HNSCC in the therapeutic context (28). In the present study, we used data retrieved from TCGA in order to explore the pathological and clinical significance of the tumour marker SCCA in HNSCC. Our analyses confirmed the broad spectrum of expression of SERPINB3/B4 across tumour types, and the high expression levels of SERPINB3/B4 in HNSCC compared to other tumours. We found that SERPINB3 mRNA levels differed significantly depending on tumour etiology (alcohol, HPV), the location of the primary tumour, tumour grade, extension to lymph nodes and extracapsular spread. Expression levels of SERPINB3 were not related to any of the genetic alterations reported to play a role in HNSCC progression (25). Interestingly, tumour SERPINB3 mRNA levels were found to be associated with potential biological determinants of tumour immunogenicity: in HPVnegative HNSCC, high levels of SERPINB3 expression were associated with a high tumour immune infiltrate score and high expression levels of the immune checkpoint modulators PD-L1 and PD-L2.

Measuring serum concentrations of SCCA constitutes a simple method that has previously been proposed to be of potential use for the diagnosis and follow-up of HNSCC patients (29-32). While the information gained from serum analysis $v s$. mRNA levels in tumours are likely different, it is interesting to consider the regulation of SERPINB3/B4 mRNA levels in HNSCC and its potential implications for the use of SCCA. Firstly, we found that high expression of SERPINB3/B4 mRNA is present in tumours other than HNSCC. Secondly, we observed that high mRNA levels of $S E R P I N B 3 / B 4$ are characteristic of less aggressive tumours in HNSCC. High SERPINB3 expression was negatively associated with tumour extension to lymph nodes and extracapsular spread, two essential pathological features that are used clinically to identify high risk HNSCC tumours that require adjuvant radiochemotherapy $(3,4)$. Both facts suggest that SCCA might not be suited for diagnostic use (33). On the other hand, the SERPINB3/B4 status of HNSCC tumours could be helpful at the time of initial assesment of tumour agressiveness. Based on our study, we propose that analyses centered on the SERPINB3 status may help physicians to adapt the follow-up of individual HNSCC patients, and help in the detection of tumour recurrence at an early stage.

The finding that HNSCC tumours with a HPV-negative status and high expression of SERPINB3 mRNA have significantly higher immune score values and higher expression levels of the genes encoding PD-L1 and PD-L2 is an important finding of the present study. Indeed, recent studies have established the clinical efficacy of checkpoint inhibitors directed against programmed cell death-1 (PD-1) and PD-L1 for the treatment of recurrent/metastatic HNSCC (6-8). Because of the cost of these treatments and their toxicity, identifying biological parameters that predict their efficacy in individual patients is required $(26,34)$. Out of the five most important parameters that define the «tumour immunogram» (26), we found that at least three are associated with the SERPINB3 status: i) the presence of an immune infiltrate; ii) high expression of the immune checkpoint modulator PD-L1, a marker associated with a better response to immunotherapy in $\operatorname{HNSCC}(7,8,35)$; iii) tumour sensitivity to immune effectors, such as Ifn- $\gamma$, as shown here with the IFNG signature reported by Chow et al. (27). Interestingly, similarly to our observations with SERPINB3, high expression levels of another tumour marker, Alpha-foetoprotein (AFP), were recently found in hepatocellular carcinoma cells with increased expression of PD-L1 (36). Based on our results and those of other recent studies on SERPINB3/B4 $(15,19)$, we propose that further clinical studies are warranted to examine whether SCCA would be useful for predicting the efficacy of immune checkpoint inhibitors in HNSCC patients.

\section{Acknowledgements}

The Authors gratefully acknowledge the contribution of the patients and researchers involved in TCGA. Work in the Authors laboratory is supported by the CHU Amiens Picardie and Ligue contre le Cancer, comité de la Somme.

\section{References}

1 Argiris A, Karamouzis MV, Raben D and Ferris RL: Head and neck cancer. Lancet 371: 1695-1709, 2008.

2 Bose P, Brockton NT and Dort JC: Head and neck cancer: from anatomy to biology. Int J Cancer 133: 2013-2023, 2013. 
3 Bernier J, Domenge C, Ozsahin M, Matuszewska K, Lefèbvre JL, Greiner RH, Giralt J, Maingon P, Rolland F, Bolla M, Cognetti F, Bourhis J, Kirkpatrick A and van Glabbeke M: Postoperative irradiation with or without concomitant chemotherapy for locally advanced head and neck cancer. $\mathrm{N}$ Engl J Med 350: 1945-1952, 2004

4 Bernier J, Cooper JS, Pajak TF, van Glabbeke M, Bourhis J, Forastiere A, Ozsahin EM, Jacobs JR, Jassem J, Ang KK and Lefèbvre JL: Defining risk levels in locally advanced head and neck cancers: a comparative analysis of concurrent postoperative radiation plus chemotherapy trials of the EORTC (\#22931) and RTOG (\# 9501). Head Neck 27: 843-850, 2005.

5 Bernier J, Bentzen SM and Vermorken JB: Molecular therapy in head and neck oncology. Nat Rev Clin Oncol 6: 266-277, 2009.

6 Seiwert TY, Burtness B, Mehra R, Weiss J, Berger R, Eder JP, Heath K, McClanahan T, Lunceford J, Gause C, Cheng JD and Chow LQ: Safety and clinical activity of pembrolizumab for treatment of recurrent or metastatic squamous cell carcinoma of the head and neck (KEYNOTE-012): an open-label, multicentre, phase 1b trial. Lancet Oncol 17: 956-965, 2016.

7 Chow LQ, Haddad R, Gupta S, Mahipal A, Mehra R, Tahara M, Berger R, Eder JP, Burtness B, Lee SH, Keam B, Kang H, Muro K, Weiss J, Geva R, Lin CC, Chung HC, Meister A, DolledFilhart M, Pathiraja K, Cheng JD and Seiwert TY: Antitumor activity of pembrolizumab in biomarker-unselected patients with recurrent and/or metastatic head and neck squamous Cell Carcinoma: Results from the Phase Ib KEYNOTE-012 Expansion Cohort. J Clin Oncol 34: 3838-3845, 2016.

8 Ferris RL, Blumenschein G Jr, Fayette J, Guigay J, Colevas AD, Licitra L, Harrington K, Kasper S, Vokes EE, Even C, Worden F, Saba NF, Iglesias Docampo LC, Haddad R, Rordorf T, Kiyota N, Tahara M, Monga M, Lynch M, Geese WJ, Kopit J, Shaw JW and Gillison ML: Nivolumab for recurrent squamous-cell carcinoma of the head and neck. N Engl J Med 375: 1856-1867, 2016.

9 Sun Y, Sheshadri N and Zong WX: SERPINB3 and B4: From biochemistry to biology. Semin Cell Dev Biol 62: 170-177, 2017.

10 Uemura Y, Pak SC, Luke C, Cataltepe S, Tsu C, Schick C, Kamachi Y, Pomeroy SL, Perlmutter DH and Silverman GA: Circulating serpin tumor markers SCCA1 and SCCA2 are not actively secreted but reside in the cytosol of squamous carcinoma cells. Int J Cancer 89: 368-377, 2000.

11 Suminami Y, Nagashima S, Murakami A, Nawata S, Gondo T, Hirakawa H, Numa F, Silverman GA and Kato H: Suppression of a squamous cell carcinoma (SCC)-related serpin, SCC antigen, inhibits tumor growth with increased intratumor infiltration of natural killer cells. Cancer Res 61: 1776-1780, 2001.

12 Ahmed ST and Darnell JE Jr.: Serpin B3/B4, activated by STAT3, promote survival of squamous carcinoma cells. Biochem Biophys Res Commun 378: 821-825, 2009.

13 Ullman E, Pan JA and Zong WX: Squamous cell carcinoma antigen 1 promotes caspase-8-mediated apoptosis in response to endoplasmic reticulum stress while inhibiting necrosis induced by lysosomal injury. Mol Cell Biol 31: 2902-2919, 2011.

14 Nakashima T, Yasumatsu R, Kuratomi Y, Masuda M, Kuwano T, Toh S, Umezaki T, Cataltepe S, Silverman GA and Komune S: Role of squamous cell carcinoma antigen 1 expression in the invasive potential of head and neck squamous cell carcinoma. Head Neck 28: 24-30, 2006.

15 Catanzaro JM, Sheshadri N, Pan JA, Sun Y, Shi C, Li J, Powers RS, Crawford HC and Zong WX: Oncogenic Ras induces inflammatory cytokine production by upregulating the squamous cell carcinoma antigens SerpinB3/B4. Nat Commun 5: 3729, 2014.

16 Sheshadri N, Catanzaro JM, Bott AJ, Sun Y, Ullman E, Chen EI, Pan JA, Wu S, Crawford HC, Zhang J and Zong WX: SCCA1/SERPINB3 promotes oncogenesis and epithelialmesenchymal transition via the unfolded protein response and IL6 signaling. Cancer Res 74: 6318-6329, 2014.

17 Urra H, Dufey E, Avril T, Chevet E and Hetz C: endoplasmic reticulum stress and the hallmarks of cancer. Trends Cancer 2: 252-262, 2016

18 Avril T, Vauléon E and Chevet E: Endoplasmic reticulum stress signaling and chemotherapy resistance in solid cancers. Oncogenesis 6: e373, 2017.

19 Riaz N, Havel JJ, Kendall SM, Makarov V, Walsh LA, Desrichard A, Weinhold N and Chan TA: Recurrent SERPINB3 and SERPINB4 mutations in patients who respond to antiCTLA4 immunotherapy. Nat Genet 48: 1327-1329, 2016.

20 The Cancer Genome Atlas Network: Comprehensive genomic characterization of head and neck squamous cell carcinomas. Nature 517: 576-582, 2015.

21 Cerami E, Gao J, Dogrusoz U, Gross BE, Sumer SO, Aksoy BA, Jacobsen A, Byrne CJ, Heuer ML, Larsson E, Antipin Y, Reva B, Goldberg AP, Sander C and Schultz N: The cBio cancer genomics portal: an open platform for exploring multidimensional cancer genomics data. Cancer Discov 2: 401-404, 2012.

22 Gao J, Aksoy BA, Dogrusoz U, Dresdner G, Gross B, Sumer SO, Sun Y, Jacobsen A, Sinha R, Larsson E, Cerami E, Sander C and Schultz N: Integrative analysis of complex cancer genomics and clinical profiles using the cBioPortal. Sci Signal 6: pl1, 2013.

$23 \mathrm{Mi} \mathrm{H}$, Muruganujan A, Casagrande JT and Thomas PD: Largescale gene function analysis with the PANTHER classification system. Nat Protoc 8: 1551-1566, 2013.

24 Yoshihara K, Shahmoradgoli M, Martínez E, Vegesna R, Kim H, Torres-Garcia W, Treviño V, Shen H, Laird PW, Levine DA, Carter SL, Getz G, Stemke-Hale K, Mills GB and Verhaak RG: Inferring tumour purity and stromal and immune cell admixture from expression data. Nature Commun 4: 2612, 2013.

25 Dotto GP and Rustgi AK: Squamous Cell Cancers: A unified perspective on biology and genetics. Cancer Cell 29: 622-637, 2016.

26 Blank CU, Haanen JB, Ribas A and Schumacher TN: Cancer Immunology. The "cancer immunogram". Science 352: 658-660, 2016.

27 Chow LQ, Mehra R, Haddad RI, Mahipal A, Weiss J and Berger $\mathrm{R}$ : Biomarkers and response to pembrolizumab (pembro) in recurrent/metastatic head and neck squamous cell carcinoma (R/M HNSCC). J Clin Oncol 34: 6010, 2016.

28 Kang H, Kiess A and Chung CH: Emerging biomarkers in head and neck cancer in the era of genomics. Nat Rev Clin Oncol 12: 11-26, 2015.

29 Guerra EN, Rêgo DF, Elias ST, Coletta RD, Mezzomo LA, Gozal D and De Luca Canto G: Diagnostic accuracy of serum biomarkers for head and neck cancer: A systematic review and meta-analysis. Crit Rev Oncol Hematol 101: 93-118, 2016.

30 Adel M, Tsao CK, Wei FC, Chien HT, Lai CH, Liao CT, Wang HM, Fan KH, Kang CJ, Chang JT and Huang SF: Preoperative SCC antigen, CRP serum levels, and lymph node density in oral squamous cell carcinoma. Medicine (Baltimore) 95: e3149, 2016. 
31 Travassos DC, Fernandes D, Massucato EMS, Navarro CM and Bufalino A: Squamous cell carcinoma antigen as a prognostic marker and its correlation with clinicopathological features in head and neck squamous cell carcinoma: Systematic review and meta-analysis. J Oral Pathol Med, 2017. doi: 10.1111/jop.12600. [Epub ahead of print]

32 Chen IH, Liao CT, Wang HM, Huang JJ, Kang CJ and Huang SF: Using SCC antigen and CRP levels as prognostic biomarkers in recurrent oral cavity squamous cell carcinoma. PLoS One 9: e103265, 2014.

33 Srivastava S, Reid BJ, Ghosh S and Kramer BS: Research needs for understanding the biology of overdiagnosis in cancer screening. J Cell Physiol 231: 1870-1875, 2016.

34 Chen K, Ye H, Lu XJ, Sun B and Liu Q: Towards in silico prediction of the immune-checkpoint blockade response. Trends Pharmacol Sci 38: 1041-1051, 2017.
35 Müller T, Braun M, Dietrich D, Aktekin S, Höft S, Kristiansen G, Göke F, Schröck A, Brägelmann J, Held SAE, Bootz F and Brossart P: PD-L1: a novel prognostic biomarker in head and neck squamous cell carcinoma. Oncotarget 8: 52889-52900, 2017.

36 Calderaro J, Rousseau B, Amaddeo G, Mercey M, Charpy C, Costentin C, Luciani A, Zafrani ES, Laurent A, Azoulay D, Lafdil $\mathrm{F}$ and Pawlotsky JM: Programmed death ligand 1 expression in hepatocellular carcinoma: Relationship With clinical and pathological features. Hepatology 64: 2038-2046, 2016.

Received December 12, 2017

Revised January 8, 2018

Accepted January 12, 2018 